\title{
A morphometric analysis of Torpedo synaptic vesicles isolated by iso-osmotic sucrose gradient separation
}

\author{
G.Q. Fox, D. Kötting and G.H.C. Dowe \\ Max-Planck-Institut für Biophysikalische Chemie, Göttingen (F.R.G.)
}

(Accepted 28 February 1989)

Key words: Vesicle; Sucrose gradient; Zonal centrifugation; Acetylcholine; Torpedo electric organ; Morphometric analysis

\begin{abstract}
The presynaptic terminal vesicle population of Torpedo electric organ is heterogeneous in size, consisting of two prominent subpopulations that comprise $80 \%$ of the total. The use of standard iso-osmotic sucrose gradients with zonal centrifugation to isolate vesicle fractions that co-localize with the acetylcholine $(\mathrm{ACh})$ peak results in the recovery of: (1) $10 \%$ of the total estimated vesicle population; and (2) a single $68-\mathrm{nm}$ diameter vesicle size class. The whereabouts of the major 90 -nm subclass, which accounts for $60 \%$ of the total terminal population and which has long been considered to represent the resident ACh population, has been investigated. Assuming this subclass to have undergone severe osmotic stress, the effects of hypo- and hyper-osmotic salines, buffers and fixatives were examined and found to produce only negligible changes on vesicle size. Isolation of vesicles by hypo-osmotic shocking of synaptosomes purified on a Ficoll gradient, however, resulted in a reasonable approximation of the in situ distribution. As the iso-osmotic sucrose gradient procedure utilizes frozen blocks of electric tissue, this step is suspected of being involved in the loss, perhaps because of the slow freezing rates employed. These findings indicate that the $90 \mathrm{~nm}$ subclass is lost rather than transformed during isolation by sucrose gradient separation and that dimensionally, the cholinergic vesicle is a constant-sized and relatively stable structure.
\end{abstract}

\section{INTRODUCTION}

The electric organ of Torpedo has long been a favored system for the study of cholinergic function with a major attraction being the possibility of establishing correlations between synaptic vesicle size and function. Zimmermann and Whittaker ${ }^{26.27}$, for example, first showed that when in situ preparations of electric organ were exposed to long term, low frequency electrical stimulation, a subclass of vesicle approximately $25 \%$ smaller in diameter resulted. If these preparations were then allowed to recover, the original, larger subclass reappeared, replacing the smaller. Correlative biochemical studies on fractions of isolated vesicles (prepared by sucrose density separation on zonal rotors) led to the conclusion that both of these subclasses were cholinergic $(\mathrm{ACh})$ and that the large subclass $(90 \mathrm{~nm})$ represented the fully loaded vesicle (subsequently termed $\left.\mathrm{VP}_{1}\right)$ whereas the smaller $(70 \mathrm{~nm})\left(\mathrm{VP}_{2}\right)$, more dense vesicle was only partially filled ${ }^{19,28-30}$. Interpreted in the light of the vesicle hypothesis, this smaller class was seen as representing the recently stimulated, recycling vesicle pool, that initially originated from the larger, resting state population ${ }^{24}$.

This interpretation has been based, in part, on a conclusion from morphometric analysis that the resting terminal contains a single $90 \mathrm{~nm}$ vesicle class $^{14.16,26}$. This point has recently come to be questioned from several sources. (1) Morphologic: multiple-sized subclasses of vesicles have been claimed to be present in resting terminals ${ }^{5,6}$. This has come about because of a differing interpretation of the histogram curves that describe size distributions. It is argued that curves of negative (left)-skew are indicative of multiple size classes whereas normal,

Correspondence: G.Q. Fox, AbG. 161, Max-Planck-Institut für Biophysikalische Chemie, 3400 Göttingen, F.R.G. 
positive (right)-skew, and log-normal curves represent single size populations ${ }^{6}$. Negative-skewed distributions, have, so far, only been found in vesicle populations from the terminals of Torpedo electric organ ${ }^{5}$. These distributions, in addition to having a $90 \mathrm{~nm}$ mode, often have discontinuities on their left slopes, and in particular one at $70 \mathrm{~nm}$, which is further indication of, at least, two subpopulations. These two are dimensionally similar to the $\mathrm{VP}_{1}$ and $\mathrm{VP}_{2}$ populations described above $\mathrm{e}^{5,6}$. (2) Physiology: electrophysiological studies have indicated that the smaller $(70 \mathrm{~nm})$ subclass of vesicle is actually present in resting organ and is merely 'uncovered' during the course of stimulation ${ }^{11}$. (3) Biochemistry: ACh Torpedo vesicle heterogeneity consisting of 3 biochemically defined subclasses has been verified in the resting electric organ. These 3 classes were found to be represented by vesicle populations of the approximate same size of $60-65 \mathrm{~nm}^{10,17}$.

These varied demonstrations of the presence of a smaller ACh subclass of vesicle in the terminals of resting electric organ challenge the claim that this subclass represents a temporary recycling population that manifests itself only during organ discharge.

An additional point of interest from the studies of Kiene and Stadler ${ }^{10.17}$ was their failure to isolate a 90-nm vesicle subclass using sucrose gradient separation. And indeed, examination of distributions from earlier studies are not convincing in the demonstration that the in situ distribution can be faithfully reproduced by this means of isolation (for example, compare distribution curves from Sheridan et al. ${ }^{16}$; Zimmermann and Denston ${ }^{29}$; Ohsawa et al. ${ }^{15}$; and Giompres et al. $^{7}$ ).

These comments point to an alternative possibility that the $\mathrm{ACh}$ vesicle is really represented by the smaller $70-\mathrm{nm}$ subclass and that the $90-\mathrm{nm}$ subclass has, to date, escaped biochemical definition. The objective of this study, therefore, was to morphometrically verify the success of the iso-osmotic sucrose gradient method of vesicle isolation in recovering the suspected ACh-containing 90-nm vesicle population.

\section{MATERIALS AND METHODS}

\section{Animals}

Adult Torpedo marmorata were obtained from the
Institut de Biologie Marine, Arcachon, France. The animals were kept alive in Göttingen in artificial sea water aquaria at $18{ }^{\circ} \mathrm{C}$ until use.

\section{Specimen preparation}

All animals were anesthetized with $0.05 \%(\mathrm{w} / \mathrm{v})$ ethyl $m$-aminobenzoate methansulfonate (MS-222) (Sigma) in sea water. They were then killed by spinal section and the entire electric organs removed by blunt dissection and further cut into 20 -g blocks for storage in liquid nitrogen.

Iso-osmotic fractionation and gradient preparation

Vesicles were isolated from extracts of frozen electric organ on a continuous (0.2-0.85 $\mathrm{M}$ sucrose) iso-osmotic (approx. $850 \mathrm{mOsm}$ ) sucrose- $\mathrm{NaCl}$ gradient with a $1.45-\mathrm{M}$ sucrose cushion ${ }^{3,15,23}$. Approximately $50 \mathrm{~g}$ of frozen electric organ was crushed to a fine powder and the vesicles, soluble protein and small membrane fragments extracted at $4{ }^{\circ} \mathrm{C}$ in 0.4 $\mathrm{M} \mathrm{NaCl}$ containing $10 \mathrm{mM}$ Tris- $\mathrm{HCl}$ buffer and 1 $\mathrm{mM}$ EGTA at $\mathrm{pH}$ 7.0. The suspension was passed through 4 layers of cheese cloth and the filtrate centrifuged for $30 \mathrm{~min}$ at $12,000 \mathrm{~g}(10,500 \mathrm{rpm})$ on a Sorvall SS 34 rotor. The supernatant $\left(S_{12}\right)$ was then layered on a sucrose density gradient and centrifuged in a zonal rotor (Beckman $\mathrm{Ti} 60$ ) for $3 \mathrm{~h}$ at $130,000 \mathrm{~g} / \mathrm{avg}$. $(50,000 \mathrm{rpm})$. Samples were collected for $\mathrm{ACh}$ and refractive indices determinations. Acetylcholine was assayed using the leech bioassay system ${ }^{3,23}$. Shoulders on the rising and falling slopes of the curve of recovered $\mathrm{ACh}$ indicated the relative positions of $\mathrm{VP}_{0}$ and $\mathrm{VP}_{2}$ vesicles, respectively ${ }^{24,30}$.

\section{Morphological preparation of zonal gradient frac- tions}

Samples of all gradient fractions between approximately $1 \%$ of the peak $\mathrm{ACh}$ amount were collected for morphological analysis. One $\mathrm{ml}$ of fraction was prepared for electron microscopy by adding $1 \mathrm{ml}$ of 4\% glutaraldehyde in $0.3 \mathrm{M}$ sodium cacodylate to produce a final concentration of $2 \%$ glutaraldehyde buffered at $\mathrm{pH}$ 7.2. These samples were allowed to fix at $4{ }^{\circ} \mathrm{C}$ for $12 \mathrm{~h}$ or longer. Following primary fixation, $4 \%$ osmium tetroxide $\left(\mathrm{OsO}_{4}\right)$ in $0.3 \mathrm{M}$ sodium cacodylate was added to produce a $1 \%$ working solution. This was allowed to fix for an additional $1 \mathrm{~h}$ at $4{ }^{\circ} \mathrm{C}$. The samples were then 
layered on $0.45-\mu \mathrm{m}$ Millipore filters over a circular area of $5 \mathrm{~mm}$ diameter with the use of gentle vacuum. Each was then overlaid with a 1-3\% solution of warm Agar, also with the aid of vacuum. Samples were bisected, dehydrated in ethanol and embedded in Epon 812 using propylene oxide as a transferral agent.

Synaptosomal and isolated vesicles prepared on a Ficoll gradient

Preparations of synaptosomes and isolated vesicles were kindly provided by Dr. J. Rylett using the method of Ducis and Whittaker ${ }^{4}$ based on a modification from Israel et al. ${ }^{9}$. Fresh electric organ (150 g) was finely minced with a scissors in $400 \mathrm{ml}$ of Torpedo Ringer's (in mM): $\mathrm{NaCl} 200, \mathrm{KCl} 8, \mathrm{MgCl}_{2}$ $1.8, \mathrm{CaCl}_{2} 3.4$, Tris $10, \mathrm{NaHCO}_{3} 5$, glucose 5.5, urea 300 , sucrose 100 and stirred for $30 \mathrm{~min}$. This was then passed through several layers of muslin and increasingly finer gauge stainless-steel grids and the resulting filtrate centrifuged at $6000 \mathrm{~g} \mathrm{(7000} \mathrm{rpm)} \mathrm{for}$ $20 \mathrm{~min}$ in an SS 34 rotor. The pellet was resuspended in $36 \mathrm{ml}$ of Torpedo Ringer's and $6 \times 6 \mathrm{ml}$ was placed on a Ficoll/Torpedo Ringer's gradient of the following composition: $8 \mathrm{ml}$ of $2 \% ; 12 \mathrm{ml}$ of $8 \%$ and $12 \mathrm{ml}$ of $12 \%$. This was spun on a SW 28 rotor at 50,000 g/avg. $(23,000 \mathrm{rpm})$ for $45 \mathrm{~min}$. The fractions were collected using a pipette. The $2-8 \%$ Ficoll fraction interface was diluted to $250 \mathrm{ml}$ in Torpedo Ringer's and represented the synaptosomal fraction.

Vesicles were isolated from this fraction by first centrifuging at $31,800 \mathrm{~g} / \mathrm{avg}$. $(20,000 \mathrm{rpm})$ for $30 \mathrm{~min}$ in a $\mathrm{Ti} 45$ rotor. The pellet was diluted to $300 \mathrm{ml}$ in a $20 \mathrm{mM}$ imidazole- $\mathrm{HCl}$ buffer containing $0.1 \mathrm{mM}$ $\mathrm{MgCl}_{2}$ at $\mathrm{pH} 8.5$, stirred for $90 \mathrm{~min}$ and further diluted with $3 \mathrm{M} \mathrm{KCl}$ to give a final concentration of $0.3 \mathrm{M}$. This was stirred for an additional $20 \mathrm{~min}$ and then centrifuged in a $\mathrm{Ti} 45$ rotor at $31,800 \mathrm{~g} / \mathrm{avg}$. $(20,000 \mathrm{rpm})$ for $30 \mathrm{~min}$. The vesicles, located in the supernatant, are collected by centrifugation in the $\mathrm{Ti}$ 75 rotor at $163,000 \mathrm{~g} / \mathrm{avg}$. $(50,000 \mathrm{rpm})$ for $2 \mathrm{~h}$.

Synaptosomes and isolated vesicles were prepared for electron microscopy as described above.

\section{Osmotic experiments}

For these experiments $\mathbf{V P}_{0}, \mathbf{V P}_{1}$ and $\mathbf{V P}_{2}$ vesicle classes from a standard iso-osmotic zonal preparation (as described above) were identified by their
ACh and ATP content and their refractive indices. Samples of each of these classes were placed in Torpedo Ringer's solution and the osmolarities slowly readjusted to $200,400,600,800,1300$ and 2100 mOsm by addition of either $\mathrm{H}_{2} \mathrm{O}(200-600)$ or urea (1300-2100). The samples were allowed to equilibrate for $1 \mathrm{~h}$ at $4{ }^{\circ} \mathrm{C}$. They were then fixed in a glutaraldehyde, $\mathrm{H}_{2} \mathrm{O}$ or sodium cacodylate fixative of equivalent osmolarity. For 200- and 400-mOsm solutions, this amounted to 2 and $4 \%$ glutaraldehyde in $\mathrm{H}_{2} \mathrm{O}$, respectively. Thereafter, sodium cacodylate of increasing molar strength was added to $4 \%$ glutaraldehyde to achieve the desired osmolarities. The samples were allowed to fix for $12 \mathrm{~h}$ at $4{ }^{\circ} \mathrm{C}$. Four percent $\mathrm{OsO}_{4}$ in $\mathrm{H}_{2} \mathrm{O}$ was added to give a postfixation solution of $1 \% \mathrm{OsO}_{4}$. The samples were then centrifuged at $114,000 \mathrm{~g} / \mathrm{avg}$. $(35,000 \mathrm{rpm})$ in an SW 50.1 rotor for $1 \mathrm{~h}$. The resulting pellets were resuspended in $0.4 \mathrm{M}$ sodium cacodylate and layered on $0.45-\mu \mathrm{m}$ Millipore filters, covered with Agar, dehydrated and embedded as described above.

\section{Fixation experiments}

$\mathrm{VP}_{1}$ vesicles were collected from a routine isoosmotic sucrose gradient (as previously described) and prepared for electron microscopy using primarily hypo-osmotic fixatives and buffers. The objective of these experiments was to explore the ability of the fixatives to swell the vesicles. Some samples were prepared in a primary fixative of glutaraldehyde + $\mathrm{H}_{2} \mathrm{O}$, or $0.1-0.3 \mathrm{M}$ sodium cacodylate covering an osmotic range of 400-900 mOsm.

Other samples were first incubated in sodium cacodylate buffers of 400 and $600 \mathrm{mOsm}$ for $1 \mathrm{~h}$ prior to fixation in an equivalent strength primary fixative of glutaraldehyde. Lastly, combinations of the above samples were postfixed in $\mathrm{OsO}_{4}$ solutions ranging from 400 to 800 mOsm. All samples were processed for electron microscopy as described above.

\section{Morphometric analysis}

To estimate the volume of each zonal sample a $1-\mu \mathrm{m}$ section of the $5-\mathrm{mm}$ embedded pellicle was cut and stained with Toluidine blue. An ocular micrometer of $400 \times$ magnification was used to make 10 measurements along its length and the volume computed.

Vesicle distributions were obtained by first cutting 
thin sections at a light-gold interference color (estimated at $118 \mathrm{~nm}$ ), mounting them unsupported on 150 mesh grids and counterstaining with uranyl acetate and lead citrate. Five to 10 micrographs were taken along the thickness of the sample at an uncorrected 24,000 $\times$ magnification. To approximate a systematic sampling routine the region chosen for photography was located near each grid wire at either end of a grid space. The sample positioning was done at low magnification $(3000 \times)$. Photographic prints were made at $3 \times$ magnification with a $225 \mathrm{~cm}^{2}$ reading area outlined in the center. A Zeiss particle analyser was used to count and size all vesicle profiles located therein. To be counted the object had to be circular to ovoid and membrane bound. The electron microscope was calibrated using a cross-grating replica (2160 lines $/ \mathrm{mm}$ ), the photographic enlarger using a linear rule and the particle analyzer using $\mathrm{mm}$ graph paper. From these data, size distribution curves were constructed and mean sizes and densities computed. The formula: $N_{\mathrm{v}}$ $=N_{\mathrm{a}} /(D+t)^{1}$ where $N_{\mathrm{v}}=$ number per unit volume; $N_{\mathrm{a}}=$ number per unit area; $D=$ mean diameter and $t=$ section thickness was used throughout for density computations.

\section{RESULTS}

\section{In situ control}

Presynaptic terminals from Torpedo electric organ routinely prepared in an iso-osmotic glutaraldehydesodium cacodylate fixative of 800 mOsm appear as in Fig. 1A. Vesicles appear throughout as lucent-cored, spherical structures bounded by a $5.3-6.6 \mathrm{~nm}$ plasma membrane. A histogram of their diameter distribution and a statistical summary of these data are shown in Fig. 1D (curve 1) and Table I. The distribution of their sizes takes the form of a negative skew curve with a mode at $90 \mathrm{~nm}$ and a shoulder at $70 \mathrm{~nm}$.

Isolated vesicles prepared by iso-osmotic sucrose gradient zonal centrifugation

Four zonal preparations were examined biochemically and morphometrically and the tabulated results are presented in Table I. As the 4 runs produced essentially similar results, the data from run 1 are presented as representative. Fig. 1B,C illustrates the appearance of vesicles isolated from run 1 showing (B) fraction 26 , which represents the size mode fraction, and $(C)$ fraction 30 , which is the ACh mode fraction. Measurement of vesicle membrane thickness varied between 5.3 and $6.6 \mathrm{~nm}$ throughout. Fig. 2 graphically summarizes the biochemical and morphometric findings obtained from this run. The ACh peak is located in fraction 30 at a refractive index of 1.3554 . The estimated number of vesicles/ml of fraction is highest under this peak and averages $1.26 \times 10^{11}$. This represents an approximate $10 \%$ recovery based on an estimate of $5 \times 10^{14}$ vesicles for $50 \mathrm{~g}$ of electric tissue (i.e. 100 $\mathrm{ml}$ of sample were prepared from the $50 \mathrm{~g}$ prior to separation). The mean size of recovered vesicles from all fractions was $68 \pm 4 \mathrm{~nm}$. The sizes drift between 62 and $75 \mathrm{~nm}$ across a sucrose density range of $0.600 \mathrm{M}$. There is no correlation between size and density from these data. A summary size distribution of all fractions is graphically represented in Fig. 1D (curve 2). It is seen to be a normal curve having a mode at $68 \mathrm{~nm}$. The most striking feature is the absence of a $90-\mathrm{nm}$ subclass of vesicle; the zonal peak coinciding instead with the $70-\mathrm{nm}$ shoulder of the in situ distribution (Fig. 1D). To examine whether overall shrinkage could account for this

Fig. 1. A: Torpedo electric organ presynaptic terminals in synaptic contact with the ventral electrocyte surface. The 3 terminals contain a dispersed population of clear, spherical, membrane-bound vesicles. B and C: appearance of vesicles isolated by iso-osmotic sucrose separation and zonal centrifugation. B is a sample from zonal fraction 26 and represents the size mode from this particular run. C comes from zonal fraction 30, the ACh mode fraction of the same run. All circular to ovoid, membrane-bound profiles were counted and measured for purpose of analysis. Note that these vesicles are also spherical but appear generally smaller than those from the intact terminals above. D: size distributions of the vesicles from in situ and isolated preparations are illustrated by these histograms. Curve 1 is the distribution of the in situ terminal population and curve 2 of the isolated vesicles as prepared by iso-osmotic sucrose density centrifugation. The smoothed data of curve 1 comes from Fox et al. ${ }^{5}$. It illustrates the negative-skew form of curve typical for vesicles from Torpedo electric organ. Note the mode at $90 \mathrm{~nm}$ and the shoulder at $70 \mathrm{~nm}$, which indicates the relative position of the two dominant subclass populations. Curve 2 represents a summary plot of the size distributions from 27 continuous zonal fractions that span the recovered $\mathrm{ACh}$ peak. It is a normal curve with mean and mode at $70 \mathrm{~nm}$ and with no indication of subpopulations. Bar $=500 \mathrm{~nm}$. 

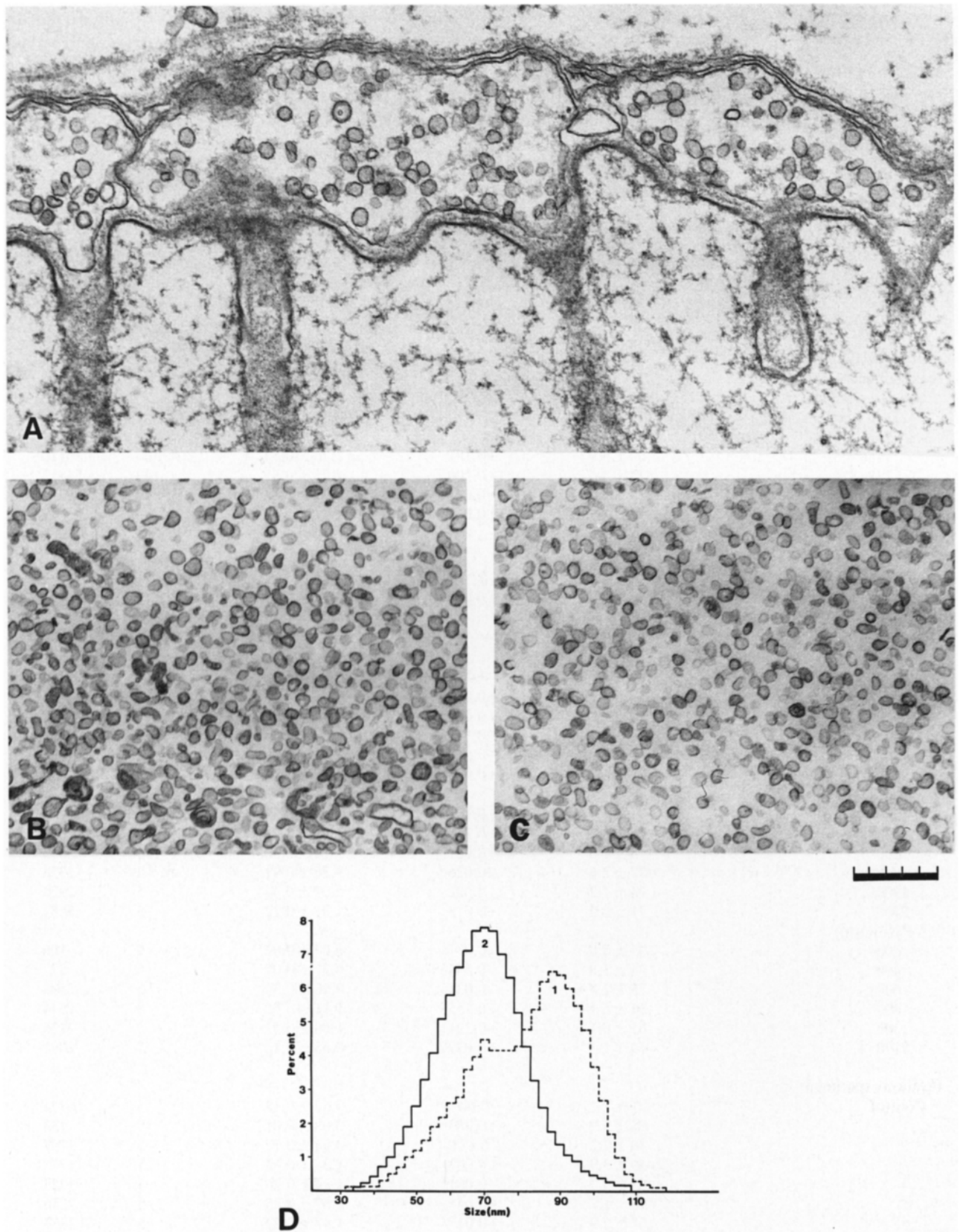
absence, two types of experiments were conducted.

Osmotic perturbation of vesicles isolated from isoosmotic sucrose zonal gradients

In these experiments, iso-osmotically isolated
$\mathrm{VP}_{1}, \mathrm{VP}_{0}$ and $\mathrm{VP}_{2}$ vesicles were suspended in Torpedo Ringer's solution and the osmolarity then slowly adjusted to ranges between 200 and 2100 mOsm. The results of these experiments show that the $\mathrm{VP}_{1}$ vesicles do not respond to changes in

\section{TABLE I}

Statistical analysis of isolated vesicles from Torpedo electric organ

Density values are per $\mu \mathrm{m}^{3}$ of terminal or per $\mathrm{ml}$ of fraction $\left(\times 10^{11}\right) ; \mathrm{n} . \mathrm{d} .=$ not determined. $n=$ number of micrographs.

\begin{tabular}{|c|c|c|c|c|c|}
\hline & $\begin{array}{l}\left.\operatorname{Size}_{(\mathrm{nm})} \pm S . D .\right) \\
\end{array}$ & Skew & Density ( $\pm S . D)$. & $n$ & Counts \\
\hline $\begin{array}{l}\text { Control in situ } \\
\text { Isolated vesicles }\end{array}$ & $89 \pm 1.2$ & -1.013 & $347 \pm 73$ & 10 & 3284 \\
\hline \multicolumn{6}{|l|}{ Sucrose gradient } \\
\hline \multirow{2}{*}{\multicolumn{6}{|c|}{$\begin{array}{l}\text { Zonal fractions } \\
\text { Run }\end{array}$}} \\
\hline & & & & & \\
\hline $1(18-45)$ & $68 \pm 3.9$ & +0.181 & $1.26 \pm 0.53$ & 300 & 36116 \\
\hline $2(9-35)$ & $72 \pm 4.5$ & +0.206 & $2.23 \pm 0.87$ & 140 & 34812 \\
\hline $3(20-45)$ & $65 \pm 3.5$ & +0.025 & $1.35 \pm 0.56$ & 210 & 39753 \\
\hline $4(20-45)$ & $67 \pm 5.5$ & +0.016 & $1.51 \pm 0.66$ & 290 & 46239 \\
\hline \multicolumn{6}{|l|}{ Ficoll gradient } \\
\hline Synaptosomes & $86 \pm 1.0$ & -0.212 & $321 \pm 34$ & 28 & 4981 \\
\hline Isolated vesicles & $81 \pm 1.3$ & +0.002 & n.d. & 15 & 6491 \\
\hline \multicolumn{6}{|l|}{ Osmotic experiments } \\
\hline \multicolumn{6}{|l|}{$\mathrm{VP}_{0}(\mathrm{mOsm})$} \\
\hline 200 & $101 \pm 2.1$ & -0.037 & $0.04 \pm 0.02$ & 5 & 65 \\
\hline 400 & $81 \pm 2.3$ & +0.092 & $0.90 \pm 0.16$ & 5 & 627 \\
\hline 600 & $68 \pm 0.9$ & +0.031 & $1.10 \pm 0.35$ & 5 & 2098 \\
\hline 800 & $72 \pm 1.3$ & +0.116 & $1.39 \pm 0.30$ & 5 & 1796 \\
\hline 1300 & $67 \pm 2.0$ & +0.223 & $1.06 \pm 0.66$ & 5 & 1791 \\
\hline 2100 & $72 \pm 3.2$ & +0.098 & $0.56 \pm 0.14$ & 5 & 1691 \\
\hline \multicolumn{6}{|l|}{$\mathrm{VP}_{1}(\mathrm{mOsm})$} \\
\hline 200 & $79 \pm 4.7$ & +0.194 & $0.85 \pm 0.37$ & 5 & 618 \\
\hline 400 & $76 \pm 2.6$ & +0.158 & $1.46 \pm 0.23$ & 5 & 1156 \\
\hline 600 & $75 \pm 1.3$ & +0.021 & $3.19 \pm 0.50$ & 5 & 3059 \\
\hline 800 & $77 \pm 2.6$ & +0.026 & $3.26 \pm 0.91$ & 5 & 3333 \\
\hline 1300 & $74 \pm 1.2$ & +0.022 & $2.09 \pm 0.44$ & 5 & 2922 \\
\hline 2100 & $71 \pm 2.2$ & +0.018 & $1.21 \pm 0.22$ & 5 & 3581 \\
\hline \multicolumn{6}{|l|}{$\mathrm{VP}_{2}(\mathrm{mOsm})$} \\
\hline 200 & $95 \pm 5.0$ & +0.037 & $0.04 \pm 0.02$ & 5 & 106 \\
\hline 400 & $74 \pm 3.8$ & +0.544 & $0.37 \pm 0.07$ & 5 & 877 \\
\hline 600 & $73 \pm 2.7$ & +0.031 & $0.98 \pm 0.30$ & 5 & 2592 \\
\hline 800 & $74 \pm 5.1$ & +0.155 & $1.41 \pm 0.66$ & 5 & 1544 \\
\hline 1300 & $65 \pm 0.1$ & +0.221 & $1.68 \pm 0.44$ & 5 & 2126 \\
\hline 2100 & $69 \pm 1.3$ & +0.079 & $0.65 \pm 0.12$ & 5 & 2081 \\
\hline \multicolumn{6}{|l|}{ Fixation experiment } \\
\hline Control & $77 \pm 1.2$ & -0.006 & $0.57 \pm 0.18$ & 5 & 1774 \\
\hline 1 & $75 \pm 5.9$ & +0.383 & $0.08 \pm 0.01$ & 5 & 133 \\
\hline 2 & $76 \pm 2.2$ & -0.021 & $0.94 \pm 0.17$ & 5 & 1757 \\
\hline 3 & $69 \pm 3.9$ & +0.003 & $1.24 \pm 0.30$ & 5 & 1775 \\
\hline 4 & $69 \pm 0.1$ & +0.001 & $1.84 \pm 0.26$ & 5 & 1725 \\
\hline 5 & $68 \pm 1.7$ & +0.045 & $1.67 \pm 0.35$ & 5 & 2256 \\
\hline 6 & $67 \pm 0.9$ & +0.045 & $1.47 \pm 0.24$ & 5 & 1837 \\
\hline
\end{tabular}


osmolarity. $\mathrm{VP}_{0}$ and $\mathrm{VP}_{2}$ vesicles, on the other hand, are of notably large dimension in the hypo-osmotic $200 \mathrm{mOsm}$ range but then fall to a $65-80 \mathrm{~nm}$ size thereafter (Fig. 3A). Note that the yield at 200 mOsm is very low (total counts, Table $\mathrm{I}$ ) indicating a sizeable loss of vesicles.

Combining all $\mathrm{VP}_{1}$ distributions between 400 and $1300 \mathrm{mOsm}$ (the normal working range) to determine whether an in situ distribution could be reproduced resulted in the normal curve seen in Fig. 3B. This curve has a coincident mode and mean at $75 \mathrm{~nm}$ and corresponds closely to the distribution obtained from $\mathrm{VP}_{1}$ vesicles at iso-osmolarity, despite the component osmolarities it represents.

These data indicate that: (1) $400 \mathrm{mOsm}$ is about the hypo-osmotic limit for some species of vesicles; and (2) most of the ACh vesicles are osmotically inactive under these conditions and can, therefore, be considered as structurally stable throughout a broad osmotic range, regardless of the status of their contents.

\section{Osmotic fixation of isolated $V P_{1}$ vesicles}

Fig. $3 \mathrm{C}$ shows the changes in $\mathrm{VP}_{1}$ mean diameter when prepared for electron microscopy by different fixation protocols. The control preparation (C) was processed at an iso-osmotic 800 mOsm throughout

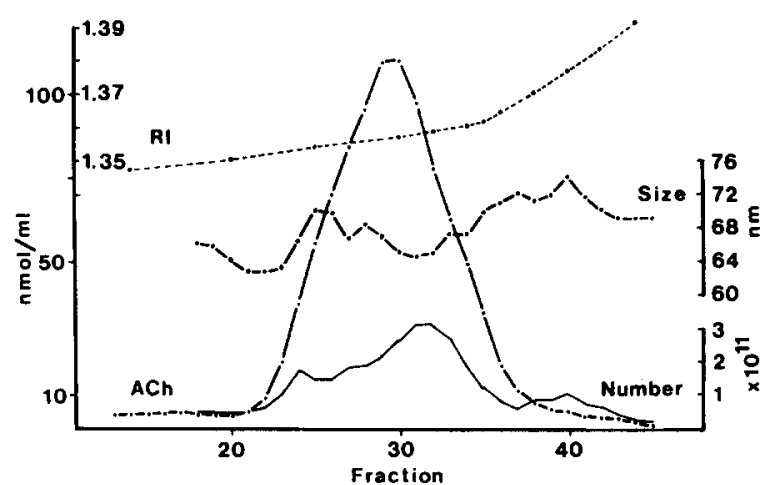

Fig. 2. This graph summarizes quantitative data obtained from one zonal run. The fractions chosen for analysis were selected according to the position of the ACh peak, here located at fraction 30. For this run, fractions 18 through 45 were evaluated morphometrically. This spans a refractive index (RI) of 1.35-1.39 which represents approximately $0.400 \mathrm{M}$ of sucrose. No correlation between density and size is evident as seen by the wandering of sizes between 62 and $75 \mathrm{~nm}(68+$ $4 \mathrm{~nm}$ ). Estimates of the number of vesicles isolated per fraction are highest for the fractions lying under the ACh peak.
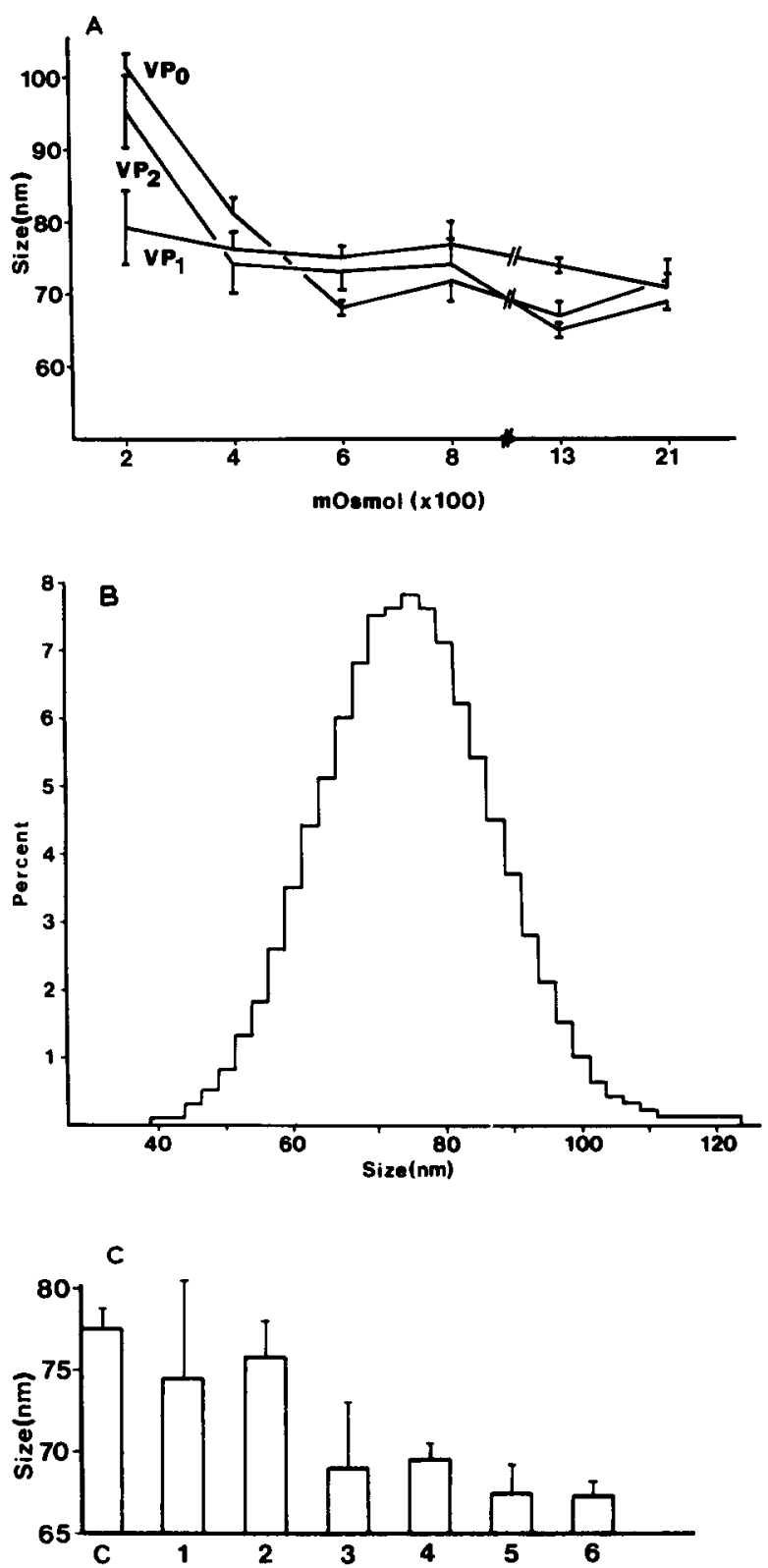

Fig. 3. Summary data of attempts to influence vesicle size by altering the osmotic conditions of the gradients and fixation media. A: results of size changes induced in biochemically isolated $\mathrm{VP}_{0}, \mathrm{VP}_{1}$ and $\mathrm{VP}_{2}$ vesicle preparations by incubation in hypo-, iso- and hyper-osmotic medias (see text for further details). $\mathrm{VP}_{1}$ vesicles are unaffected by changes in osmolarity. $\mathrm{VP}_{0}$ and $\mathrm{VP}_{2}$ vesicles have a distinctly larger mean diameter at $200 \mathrm{mOsm}$ but then become reduced to a 'standard' size thereafter. $B$ : distribution summarizes the $V P_{1}$ distributions accumulated over a $400-1300 \mathrm{mOsm}$ range (iso $=800 \mathrm{mOsm}$ ). It is a normal curve with a mean at $75 \mathrm{~nm}$. There is no indication of the existence of a 90-nm subclass from these data. $\mathrm{C}$ : variations in mean $\mathrm{VP}_{1}$ vesicle size due to different fixation regimens. In no case could a $90-\mathrm{nm}$ vesicle size be restored regardless of the degree of hypo-osmotic incubation and fixation $^{3-6}$. For further details see Table II. 
TABLE II

Osmotic strength of the fixative components used in Fig. 3

Values are mOsm.

\begin{tabular}{lllc}
\hline Experiment & Buffer & Glutaraldehyde & Osmium tetroxide \\
\hline Control & - & 800 & 800 \\
1 & - & 600 & 400 \\
2 & - & 900 & 1000 \\
3 & 600 & 600 & 600 \\
4 & 600 & 600 & 800 \\
5 & 400 & 400 & 400 \\
6 & 400 & 400 & 800 \\
\hline
\end{tabular}
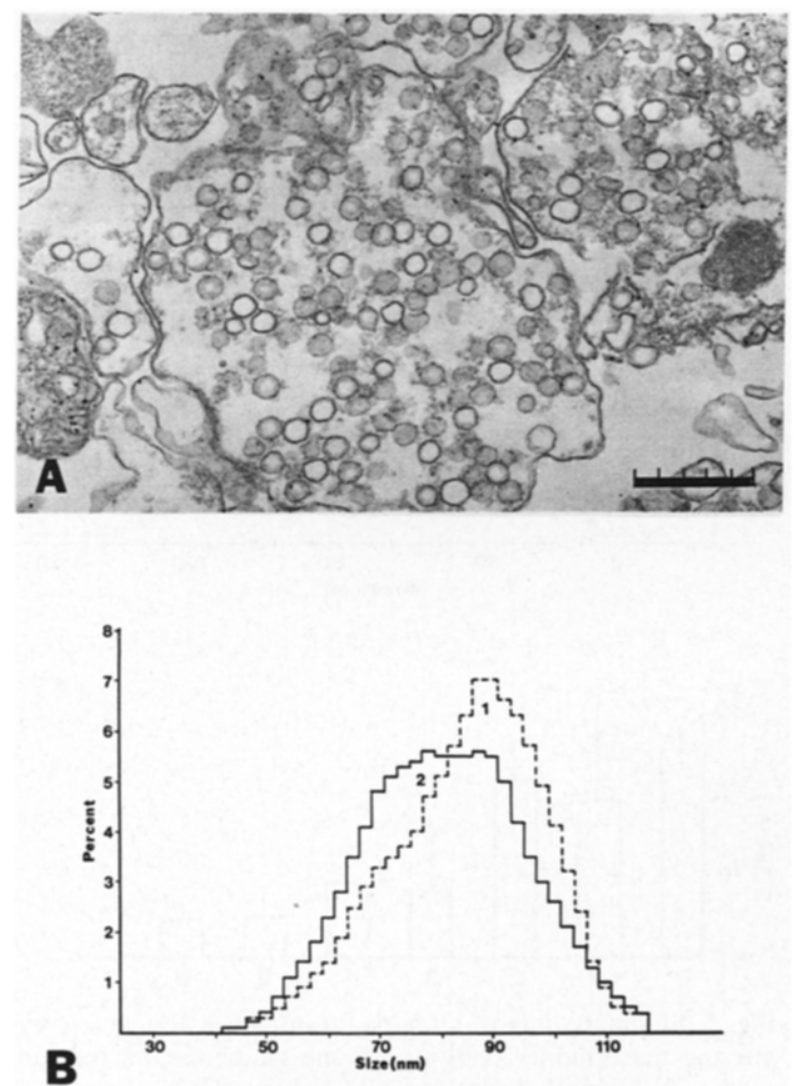

Fig. 4. A: electron micrograph of synaptosomes isolated from fresh tissue on a Ficoll gradient. Synaptosomal profiles are identified by the presence of spherical, membrane-bound vesicles. $\mathrm{Bar}=500 \mathrm{~nm}$. B: size distribution curves of vesicle populations prepared by Ficoll gradient separation. Curve 1 is the distribution obtained from the vesicle population contained in the synaptosomes of A. It is negatively skewed with a major mode at $90 \mathrm{~nm}$. Curve 2 represents the distribution of vesicles isolated from these synaptosomes by osmotic shock and centrifugation. Note that although this curve is normal in form, the mode is a plateau that extends well into the $90 \mathrm{~nm}$ range indicating a partial salvaging of this size class of vesicle. Compare these curves to those of Fig. 1D. and produced a normal curve having a mean of 77 $\mathrm{nm}$ (Table I). Lowering the primary glutaraldehyde fixative osmolarity to $600 \mathrm{mOsm}$ (Fig. $3 \mathrm{C}_{1}$ ) or increasing it to $900 \mathrm{mOsm}$ (Fig. $3 \mathrm{C}_{2}$ ) produced no change in mean size $(75$ and $76 \mathrm{~nm}$, respectively, Table I). Preincubation in a hypo-osmotic buffer (600 and $400 \mathrm{mOsm}$ ) prior to fixation (Fig. $3 \mathrm{C}_{3-6}$ ) tended to reduce the mean size by about 7 to $68 \mathrm{~nm}$ rather than enlarge it as expected. It was not possible with the combinations tested to produce a $90-\mathrm{nm}$ class of vesicle. We conclude that it is possible to alter the mean diameter of the $\mathrm{ACh}$ vesicle by changes in fixation osmolarities, but not of a magnitude that would explain a change from 90 to $68 \mathrm{~nm}$.

Torpedo electric organ synaptosomes prepared on Ficoll gradients

As all the above studies on isolated vesicles relied on the use of frozen blocks of electric organ, a Ficoll separation of synaptosomes using fresh tissue was examined. Synaptosomes prepared in this manner are shown in Fig. 4A and the distribution of their vesicle population in Fig. $4 \mathrm{~B}$ (curve 1). This distribution is representative of the typical in situ distribution (compare with Fig. 1D, curve 1). If these vesicles are isolated by osmotic shock and sized, a reasonable facsimile of the synaptosome distribution including the presence of $90 \mathrm{~nm}$ vesicles is found (Fig. 4B, curve 2). This experiment argues against the criticism that electron microscopic fixation conditions are responsible for the failure to recover the 90-nm subclass.

\section{DISCUSSION}

In this study the size distributions of vesicles isolated from Torpedo electric organ by iso-osmotic sucrose gradient separation and zonal centrifugation and spanning $\mathrm{ACh}$ peak activities have been examined. The main finding is that the dominant in situ synaptic vesicle subclass of $90 \mathrm{~nm}$ diameter is lost when employing this most conventional isolation procedure. This method was found instead to salvage a subclass of vesicles whose mean diameter is 68 $\mathrm{nm}$. The loss appears to have occurred following the freezing of the tissue and prior to zonal centrifugation. This suggests that much of the loss may be attributed to the use of frozen tissue and the slow 
freezing rates that are of necessity involved with this method.

The following considerations support this interpretation. (1) There are no morphological indications that vesicle shrinkage has occurred. One begins with a composition of 68 - and 90-nm spheres each with 6-nm plasma membranes and ends with 68-nm spheres having 6-nm membranes. (2) The use of fresh tissue with a Ficoll gradient results in the partial recovery of the 90 -nm subclass. (3) Numerical estimates of vesicle recovery indicate that sucrose gradient separation has a low recovery potential of, at best $10 \%$. And this figure includes varying unknown proportions of vesicles of non-synaptic origin. (4) Freeze fracture studies indicate: (a) that the $90-\mathrm{nm}$ subclass is retained (Dr. M. Israel, personal communication); and (b) that rapid freezing rates are directly linked to ultrastructural preservation ${ }^{18}$.

No morphological evidence could be obtained to support the idea that the osmotic properties attributed to vesicles account for the size changes involved $^{24}$. This argument derives from biophysical studies where changes in osmotic pressure are used to determine vesicular densities that, in turn, are used to estimate core volumes. From these estimates, predictions of vesicular sizes of $90 \mathrm{~nm}^{2.8}$ and $81.2 \mathrm{~nm}^{21}$ have been made for iso-osmotic Torpedo vesicles prepared as in this study. Our results as well as others ${ }^{10,13}$ show that vesicular density is not a reliable indicator for estimates of vesicular volume. We were unable to detect any significant departure from the $68 \mathrm{~nm}$ mean diameter found for vesicles positioned across an equivalent sucrose density range of $0.600 \mathrm{M}$. Nor were we able to induce significant size changes by exposing vesicles to different saline, buffer and fixation osmolarities. Furthermore, there was no indication of either vesicular collapse (the $68-\mathrm{nm}$ vesicles remain spherical in appearance) or membrane thickening (the

\section{REFERENCES}

1 Abercrombie, M., Estimation of nuclear population from microtomic sections, Anat. Rec., 94 (1946) 239-251.

2 Breer, H., Morris, S.J. and Whittaker, V.P., A structural model of cholinergic synaptic vesicles from the electric organ of Torpedo marmorata deduced from density measurements at different osmotic pressures, Eur. J. Biochem., 87 (1978) 453-458. thickness would have to more than double to account for a 90-68 nm change). That we could recover a portion of the $90-\mathrm{nm}$ subclass by using fresh tissue on Ficoll gradients, a method that employs both osmotic shock and the same fixatives, excludes these procedural steps from further consideration. We can conclude, therefore, that dimensionally the ACh vesicles, prepared by this method, are relatively stable osmotic structures ${ }^{11,20}$.

Although the $90-\mathrm{nm}$ subclass has always been considered to represent the major vesicular store of $\mathrm{ACh}$, there is no convincing biochemical/morphological verification for this view. For example, note the differences between the skewness and mode sizes of distributions of isolated $\mathrm{ACh}$ vesicles reported by Sheridan et al. ${ }^{16}$; Zimmermann and Denston ${ }^{29}$; Ohsawa et al. ${ }^{15}$; Giompres et al. ${ }^{7}$; and Kiene and Stadler ${ }^{10}$. However, support does come from physiological/morphological studies demónstrating parallel $\mathrm{ACh}$ and 90 -nm vesicle depletion by long-term, low-frequency stimulation of the electric organ ${ }^{11}$, 26,29. These experiments have led to the most recent interpretation that the $90-\mathrm{nm}$ loss is a time rather than frequency dependent phenomenon ${ }^{11}$. This remains consistent with the idea that this subclass represents a reserve population of vesicles that is only intermittently released ${ }^{19.24}$.

The picture unfolding from this study then is at some variance with the currently held views concerning the morphological composition of the vesicle population of Torpedo electric organ terminals. It suggests that the ACh vesicle is represented by a single 68-nm subclass population, a subclass that accounts for $20 \%$ of the total vesicular population ${ }^{6}$. Vesicular recycling and refilling all take place in this population rather than in the $90-\mathrm{nm}$ subclass. The $90-\mathrm{nm}$ subclass, making up $60 \%$ of the total populatior $^{6}$, seems to have eluded attempts at isolation and remains to be biochemically and functionally characterized.

3 Dowdall, M.J., Boyne, A.F. and Whittaker, V.P., Adenosine triphosphate a constituent of cholinergic synaptic vesicles, Biochem. J., 140 (1974) 1-12.

4 Ducis, I. and Whittaker, V.P., High-affinity, sodiumgradient-dependent transport of choline into vesiculated presynaptic plasma membrane fragments from the electric organ of Torpedo marmorata and reconstitution of the solubilized transporter into liposomes, Biochim. Biophys. Acta, 815 (1985) 109-127. 
5 Fox, G.Q., Kriebel, M.E. and Kötting, D., Synaptic vesicle populations and classes of quanta in Torpedo and skate electric organ and muscle. In H. Zimmermann (Ed.), Cellular and Molecular Basis of Synaptic Transmission. NATO ASI Series, Vol. H21, Springer, Heidelberg, 1988, pp. 83-96.

6 Fox, G.Q., A morphometric analysis of synaptic vesicle distributions, Brain Research, 475 (1988) 103-117.

7 Giompres, P.E., Zimmermann, H. and Whittaker, V.P., Purification of small dense vesicles from stimulated Torpedo electric tissue by glass bead column chromatograophy, Neuroscience, 6 (1981) 765-774.

8 Giompres, P.E., Morris, S.J. and Whittaker, V.P., The water spaces in cholinergic synaptic vesicles from Torpedo measured by changes in density induced by permeating substances, Neuroscience, 6 (1981) 757-763.

9 Israel, M., Manaranche, R., Mastour-Frachon, P. and Morel, N., Isolation of pure cholinergic nerve endings from the electric organ of Torpedo marmorata, Biochem. J., 160 (1976) 113-115

10 Kiene, M.-L. and Stadler, H., Synaptic vesicles in electromotoneurones. I. Axonal transport, site of transmitter uptake and processing of a core proteoglycan during maturation, EMBO J., 6 (1987) 2209-2215.

11 Kriebel, M.E., Fox, G.Q. and Kötting, D., Effect of nerve stimulation, $\mathrm{K}^{+}$saline and hypertonic saline on classes of quanta, quantal content and synaptic vesicle size distribution of Torpedo electric organ. In H. Zimmermann (Ed.), Cellular and Molecular Basis of Synaptic Transmission. NATO ASI Series, Vol. H2I, Springer, Heidelberg, 1988, pp. 97-120.

12 Morel, N. and Meunier, F.-M., Simultaneous release of acetylcholine and ATP from stimulated cholinergic synaptosomes, $J$. Neurochem., 36 (1981) 1766-1773.

13 Morris, S.J., Schultens, H.A. and Schober, R., An osmometer model for changes in the buoyant density of chromaffin granules, Biophys. J., 20 (1977) 33-48.

14 Nickel, E. and Potter, L.T., Synaptic vesicles in freezeetched electric tissue of Torpedo, Brain Research, 23 (1970) 95-100.

15 Ohsawa, K., Dowe, G.H.C., Morris, S.J. and Whittaker, V.P., The lipid and protein content of cholinergic synaptic vesicles from the electric organ of Torpedo marmorata purified to constant composition: implications for vesicle structure, Brain Research, 161 (1979) 447-457.

16 Sheridan, M.N., Whittaker, V.P. and Israel, M., The subcellular fractionation of the electric organ of Torpedo, Z. Zellforsch., 74 (1966) 291-307.

17 Stadler, H. and Kiene, M.-L., Synaptic vesicles in electromotoneurones. II. Heterogeneity of populations is expressed in uptake properties; exocytosis and insertion of a core proteoglycan into the extracellular matrix, EMBOJ.,
6 (1987) 2217-2221.

18 Steinbrecht, R.A. and Zierold, K. (Eds.), Cryotechniques in Biological Electron Microscopy, Springer, Heidelberg, 1987, pp. 297.

19 Suszkiw, J.B., Zimmermann, H. and Whittaker, V.P., Vesicular storage and release of acetylcholine in Torpedo electroplaque synapses, $J$. Neurochem., 30 (1978) 1269 1280.

20 Van Der Kloot, W. and Spielholz, N., Effects of changes in tonicity of the extracellular solution on the size of vesicles in frog motor nerve terminals, J. Neurocytol., 16 (1987) $77-84$.

21 Wagner, J.A., Carlson, S.S. and Kelly, R.B., Chemical and physical characterization of cholinergic synaptic vesicles, Biochemistry, 17 (1978) 1199-1206.

22 Whittaker, V.P., The structure and function of cholinergic synaptic vesicles, Biochem. Soc. Transm., 12 (1984) 561576.

23 Whittaker, V.P., Essman, W.B. and Dowe, G.H.C., The isolation of pure cholinergic synaptic vesicles from the electric organs of elasmobranch fish of the family Torpedinidae, Biochem. J., 128 (1972) 833-846.

24 Whittaker, V.P. and Schmid, D.W., Model cholinergic systems: the electromotor system of Torpedo. In V.P. Whittaker (Ed.), The Cholinergic Synapse, Springer, Heidelberg, 1988, pp. 23-39.

25 Whittaker, V.P., The cellular basis of synaptic transmission: an overview. In H. Zimmermann (Ed.), Cellular and Molecular Basis of Synaptic Transmission. NATO ASI Series, Vol. H21, Springer, Heidelberg, 1988, pp. 1-23.

26 Zimmermann, H. and Whittaker, V.P., Effect of electrical stimulation on the yield and composition of synaptic vesicles from the cholinergic synapses of the electric organ of Torpedo: a combined biochemical, electrophysiological and morphological study, J. Neurochem., 22 (1974) 435450 .

27 Zimmermann, H. and Whittaker, V.P., Different recovery rates of the electrophysiological, biochemical and morphological parameters in the cholinergic synapses of the Torpedo electric organ after stimulation, J. Neurochem., 22 (1974) 1109-1114.

28 Zimmermann, H. and Whittaker, V.P., Morphological and biochemical heterogeneity of cholinergic synaptic vesicles, Nature (Lond.), 267 (1977) 633-635.

29 Zimmermann, H. and Denston, C.R., Recycling of synaptic vesicles in the cholinergic synapses of the Torpedo electric organ during induced transmitter release, Neuroscience, 2 (1977) 695-714.

30 Zimmermann, H. and Denston, C.R., Separation of synaptic vesicles of different functional states from the cholinergic synapses of the Torpedo electric organ, $\mathrm{Neu}$ roscience, 2 (1977) 715-730. 